\title{
Immune adjuvant effect of Juzentaihoto, a Japanese traditional herbal medicine, on tumor vaccine therapy in a mouse model
}

\author{
NOBUHIRO TAKENO $^{1 *}$, AKIKO INUJIMA $^{1,2^{*}}$, KANNA SHINOHARA $^{1 *}$, MIYUKI YAMADA $^{1}$, \\ NAOTOSHI SHIBAHARA ${ }^{2}$, HIROAKI SAKURAI ${ }^{1,3}$, IKUO SAIKI ${ }^{1}$ and KEIICHI KOIZUMI ${ }^{1,2^{*}}$ \\ Divisions of ${ }^{1}$ Pathogenic Biochemistry and ${ }^{2}$ Kampo Diagnostics, Institute of Natural Medicine, \\ ${ }^{3}$ Department of Cancer Cell Biology, Graduate School of Medicine and Pharmaceutical Sciences, \\ University of Toyama, Toyama 930-0194, Japan
}

Received June 19, 2015; Accepted August 10, 2015

DOI: $10.3892 /$ ijo.2015.3208

\begin{abstract}
Japanese traditional herbal medicine (Kampo) have been used to improve the general physical condition after surgery and to mitigate the side effects of radiation and chemotherapy in tumor patients. Juzentaihoto (JTT) consists of ten medical herbs, and is also called Shi-Quan-Da-BuTang in Chinese herbal medicine. Among Kampo medicines, JTT has especially gained attention as a biological response modifier. Currently, clinical trials of various tumor vaccine therapies are being performed world-wide. However, tumor antigens that are inoculated as vaccines do not have high immunogenicity; thus, it is difficult to obtain an effective therapeutic effect. Thus, it is necessary to develop a tumor vaccine adjuvant that is more potent and very safe. In the present study, we examined the efficacy of JTT as an oral adjuvant when given together with tumor vaccines. As a result, JTT enhanced the phagocytic ability of OVA antigen and the presentation ability of OVA antigen in dendritic cells in vitro. Furthermore, tumor growth was markedly decreased, and the survival period was significantly prolonged in mice inoculated with mouse lymphoma, which is expressed with tumor model antigen. In conclusion, these findings suggest that JTT can be used with tumor vaccines as an immune adjuvant.
\end{abstract}

\section{Introduction}

Currently, complementary and alternative medicine (CAM) is prevalent throughout the world, so the integration of western

Correspondence to: Dr Keiichi Koizumi, Division of Pathogenic Biochemistry, Institute of Natural Medicine, University of Toyama, Toyama 930-0194, Japan

E-mail: kkoizumi@inm.u-toyama.ac.jp

*Contributed equally

Key words: tumor vaccine, Juzentaihoto, Japanese traditional herbal medicine, immune adjuvant and oriental medicine, utilizing both properties, is increasingly required. Japanese traditional herbal medicine (Kampo), for example, Juzentaihoto (JTT), which is also called Shi-QuanDa-Bu-Tang in Chinese herbal medicine, is prescribed for various diseases, and one of its properties is strong immunepotentiating activity, such as increasing antibody production (1-7). In addition, pharmacokinetics analysis of major active compound in JTT has been reported (8-11).

Vaccines are one of the major accomplishments in the history of medicine. It is now also imperative to develop novel vaccine adjuvants with high safety and efficacy, while novel vaccines are increasingly undergoing advanced development and contributing to unmet clinical needs (12).

Thus, we investigated whether the activity of Kampo was suitable as immune adjuvant for treatment with vaccines. Recently, we performed broad-ranging analysis of the adjuvant effect of JTT on influenza vaccination in elderly people, who are a high-risk group for influenza infection, in a multicenter randomized controlled trial (9). Consequently, JTT increased and prolonged antibody production after influenza vaccination. Therefore, we clinically first achieved the establishment of a new integrative vaccine therapy using Kampo as an immune adjuvant (13).

On the other hand, a wide range of tumor vaccine preparations, which have high immunoreaction specific to a tumor-related antigen, have been tested throughout the last two decades. In fact, more than twenty clinical studies that investigated therapeutic potential of tumor-associated antigens (TAAs) in tumor patients were reported in 2013 (14).

Given the success of JTT in influenza vaccination (13), we extended the use of JTT to other types of vaccine as immune adjuvant in the present study.

Here, we report that JTT is an attractive immune adjuvant candidate for tumor vaccines because of its marked inhibitory effect on tumor growth in combination with a tumor vaccine in a mouse model experiment.

\section{Materials and methods}

Reagents. Juzentaihoto (JTT) was obtained from Tsumura \& Co. (Tokyo, Japan). JTT was prepared as a spray-dried powder of a hot water extract obtained from ten medical 
herbals in the following ratio: JP astragalus root $(3.0 \mathrm{~g})$, JP cinnamon bark (3.0 g), JP rehmannia root $(3.0 \mathrm{~g})$, JP peony root (3.0 g), JP cnidium rhizome (3.0 g), Japanese angelica root (3.0 g), JP ginseng (3.0 g), JP poria sclerotium (3.0 g), JP glycyrrhiza (1.5 g) and JP Atractylodes lancea rhizome (3.0 g). Japanese pharmacopoeia (JP) is established and published to regulate the properties and quality of drugs by the Minister of Health, Labour and Welfare after hearing the opinion of the Pharmaceutical Affairs and Food Sanitation Council (The Japanese Pharmacopoeia 16th edition). JTT has been approved by the Japanese Ministry of Health, Labor and Welfare since 1986. Chemical characterization of JTT was ensured by using three-dimensional (3D) HPLC analysis (Fig. 1). JTT was dissolved in water immediately before administration to mice.

Mice. Five-week-old female C57BL/6 mice were purchased from Sankyo Labo Service (Hamamatsu, Japan) for use in experiments from 6 to 8 weeks of age. This study was carried out in strict accordance with the recommendations in the Guide for the Care and Use of Laboratory Animals of University of Toyama. The protocol was approved by the Committee on the Ethics of Animal Experiments of University of Toyama (permission no. S-2009 INM-3). All surgery was performed under sodium pentobarbital anesthesia, and all efforts were made to minimize suffering.

Cell culture. CD8-OVA1.3 cells, a murine T-T hybridoma that is specific for OVA257-264H-2 $\mathrm{Kb}^{29}$, were maintained in Dulbecco's modified Eagle's medium (DMEM) (Life Technologies Japan Ltd., Tokyo, Japan) supplemented with $10 \%$ fetal bovine serum (FBS) (Invitrogen), and 2-ME $(50 \mu \mathrm{M}$, Invitrogen), E.G7-OVA tumor cells (H-2b; OVA-transfectant of EL4 murine thymoma cells) were maintained in RPMI-1640 medium supplemented with $10 \% \mathrm{FBS}, 2-\mathrm{ME}(50 \mu \mathrm{M})$ and G418 $(400 \mu \mathrm{g} / \mathrm{ml})$. Murine dendritic DC2.4 cells (H-2b) (T-T hybridoma against OVA $+\mathrm{H}-2 \mathrm{~Kb}$ ) were grown in complete RPMI-1640 medium supplemented with non-essential amino acid $(100 \mu \mathrm{M})$ and 2-ME $(50 \mu \mathrm{M})$. Primary DC cells were positively selected from spleen (C57BL/6 mice, H-2b) using an anti-mouse CD11c-magnetic activated cell sorting (MACS) kit, according to the protocol provided by the manufacturer (Miltenyi Biotec K.K., Tokyo, Japan). The purity of primary DC cells was analyzed by CD11c antibodies conjugated to PE (BD Biosciences, CA, USA) with a FACSCanto flow cytometer (BD Pharmingen).

Phagocytosis in DC2.4 cells. Fluorescence isothiocyanate (FITC) conjugated OVA (OVA-FITC; Life Technologies Japan Ltd.) was dissolved to a concentration of $10 \mu \mathrm{g} / \mathrm{ml}$ in balanced salt solution (BSS). Phagocytosis in DC2.4 cells was performed by modification of a previously reported method (30). In this assay, AIM-V media (Life Technologies) was used instead of growth media. Briefly, $5 \times 10^{5}$ cells/well were seeded in a 3.5-cm dish (Corning International K.K., Tokyo, Japan) and incubated with JTT $(200 \mu \mathrm{g} / \mathrm{ml})$, OVA-FITC (50 $\mu \mathrm{g} /$ $\mathrm{ml})$ and Lipofectine (20 $\mu \mathrm{g} / \mathrm{ml}$; Life Technologies) for $20 \mathrm{~h}$ at $37^{\circ} \mathrm{C}$. Vectashield mounting medium for DAPI staining (Vector Laboratories, Inc., CA, USA) was added to the cells. Florescence images were captured using a Leica TCS SP5 microscope (Leica Microsystems, Wetzlar, Germany).
Antigen presentation. The in vitro assay for antigen processing and presentation was performed as described previously (31). In brief, DC2.4 cells $\left(2 \times 10^{4}\right)$ cultured on 96-well plates were incubated with JTT $(200 \mu \mathrm{g} / \mathrm{ml})$ or the ten herbal ingredients of JTT $(100 \mu \mathrm{g} / \mathrm{ml})$ at $37^{\circ} \mathrm{C}$ for $1 \mathrm{~h}$. OVA $(50 \mu \mathrm{g} / \mathrm{ml})$ with Lipofectine $(20 \mu \mathrm{g} / \mathrm{ml})$ was added and incubated at $37^{\circ} \mathrm{C}$ for $20 \mathrm{~h}$. The cells were fixed with $0.05 \%$ glutaraldehyde and washed three times, CD8-OVA1.3 cells $\left(1 \times 10^{5}\right)$ were added to each well. After 24-h cultivation, the response of stimulated CD8-OVA1.3 cells was determined by their IL-2 secretion levels using ELISA (R\&D Systems, MN, USA).

Tumor protection assay. C57BL/6 mice were subcutaneously immunized with OVA-peptide formulatied antigens at $100 \mu \mathrm{g} / 100 \mu \mathrm{l}$ incomplete Freund adjuvant/mouse. Seven days after immunization, these mice were intradermally inoculated with E.G7-OVA cells $\left(5 \times 10^{5}\right)$. Ten mice were used for each experimental group. Tumor volume was calculated as follows: $\left(\right.$ tumor volume; $\left.\mathrm{mm}^{3}\right)=($ major axis; $\mathrm{mm}) \mathrm{x}(\text { minor axis; } \mathrm{mm})^{2}$ $x 1 / 2$. Tumor measurements were determined until the volume exceeded $1,000 \mathrm{~mm}^{3}$. Tumor-bearing mice continued treatment as indicated above after 37 days. Mice were allowed to live up to their natural death or were sacrificed when their tumor volume was $>1,000 \mathrm{~mm}^{3}$. Sacrifice was performed under sodium pentobarbital anesthesia, and all efforts were made to minimize suffering. Kaplan-Meier survival curves were plotted and statistically analyzed. Animal body weight was measured and recorded every $4 \mathrm{~d}$ during the treatment for the monitoring of the condition of the animals. JTT or water was orally administered to 10 mice ( $2 \mathrm{~g} / \mathrm{kg} / \mathrm{day})$ from day 7 to day 37 consecutively.

ELISPOT. The ELISPOT assay (R\&D Systems) was performed according to the manufacturer's instructions. Briefly, 96-well PVDF plates (Millipore, MA, USA) were coated overnight at $4^{\circ} \mathrm{C}$ with $0.1 \mathrm{mg} /$ well of purified AN-18 (anti-IFN- $\gamma$ ). The membrane on the plates was pre-wetted by adding $50 \mu \mathrm{l}$ $70 \%$ ethanol to each well, then washed and blocked for $30 \mathrm{~min}$ with complete RPMI. C57BL/6 splenocytes $\left(1 \times 10^{5}\right.$ cells/well) were seeded on the 96-well PVDF plates with OVA $(50 \mathrm{mg} / \mathrm{ml}$; Sigma-Aldrich, MO, USA) at $37^{\circ} \mathrm{C}$ for $24 \mathrm{~h}$. After staining with streptavidin-alkaline phosphatase (BD Biosciences, CA, USA), IFN- $\gamma$-secreting $\mathrm{T}$ cells in the spleens were calculated under a microscope.

ELISA. OVA solutions $(10 \mathrm{mg} / \mathrm{ml})$ were added to $96-w e l l$ ELISA plates and incubated at $4^{\circ} \mathrm{C}$ overnight. Unbound OVA on the plate was removed by washing, and the plate was further incubated with blocking reagent (Morinaga Institute of Biological Science, Inc., Yokohama, Japan) at $37^{\circ} \mathrm{C}$ for $1 \mathrm{~h}$. Dilutions of mouse serum immunized with OVA with or without the administration of JTT were added to the wells (100 $\mu \mathrm{l} /$ well) and incubated at $37^{\circ} \mathrm{C}$ for $1 \mathrm{~h}$. After washing, anti-mouse IgG1-HRP (15H6, G1 chain specific; SouthernBiotech, AL, USA) or IgG2a-HRP (HOPC-1, G2a chain specific; SouthernBiotech) was added to the wells and incubated at $37^{\circ} \mathrm{C}$ for $1 \mathrm{~h}$. These IgG1 and IgG2 levels were determined using TMBZ (3,3',5,5'-tetramethylbenzidine; Dojindo, Kumamoto, Japan) according to the manufacturer's instructions. 


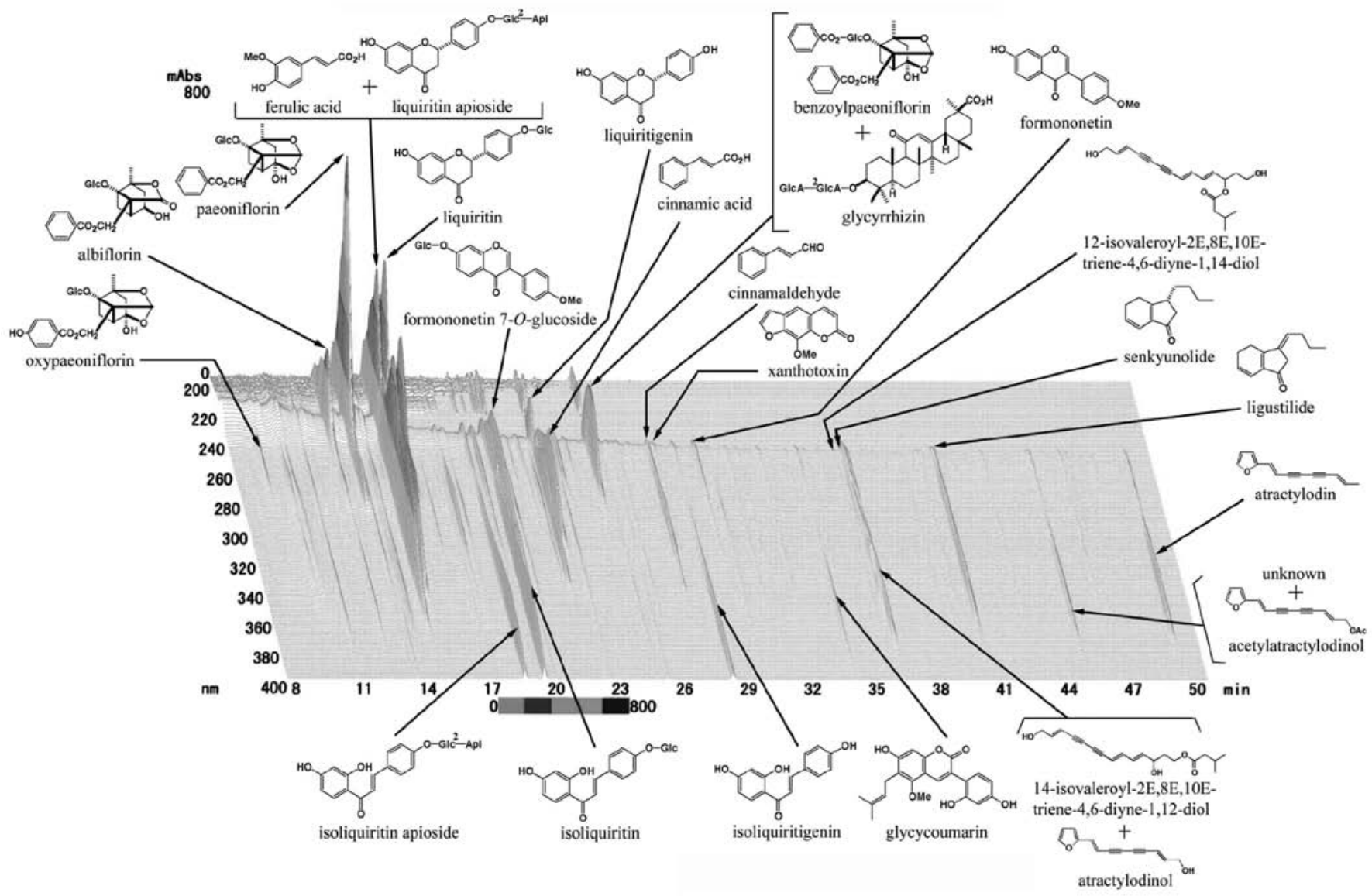

Figure 1. Three-dimensional (3D) HPLC analysis for profiling JTT.

Statistical analysis. Data are presented as the mean \pm standard deviation (SD) and were analyzed statistically by the two-tailed Student's t-test. Survival was analyzed by the Kaplan-Meier method and compared between groups by use of the log-rank test. Analyses were performed by means of JMP (Version 11, SAS Institute Inc., NC, USA). Results with $\mathrm{P}<0.05$ were considered significant and similar results were obtained from three independent experiments.

\section{Results}

Increasing effects of JTT and its herbal ingredients on antigen presentation to MHC class I pathway in DC2.4 cells and primary DC cells. Presently, JTT is manufactured according to the Ethical Kampo Medicine Drug GMP regulations and the self-imposed regulations of the Japan Kampo-Medicine Manufacturers Association. The quality of its main component was guaranteed through three-dimensional (3D) HPLC analysis (Fig. 1).

To determine JTT ability in the uptake of antigen phagocytosis, murine dendritic DC2.4 cells were incubated with soluble OVA-FITC. DC2.4 cells were fixed and labeled with DAPI, a nucleotide marker. DC2.4 cells slightly phagocytosed OVA-FITC in the absence of JTT (Fig. 2A). On the other hand, the numbers of phagocytosis of DC2.4 cells with OVA-FITC significantly increased 3.3-fold in the presence of JTT (Fig. 2B and $\mathrm{C}$ ). However, no change of the fluorescence intensity per single DC was observed by using of image analyzer (data not shown). Therefore, JTT may not improve the efficacy of phagocytosis by single DC.

Owing to the increase of phagocytosis, we next examined whether JTT introduced OVA antigen into the MHC class I presentation pathway in dendritic cells. No antigen presentation was detected by $\mathrm{T}$ cells alone or by $\mathrm{T}$ cells cocultured with DC 2.4 cells not loaded with OVA and preincubated with JTT. DC2.4 cells preincubated with JTT efficiently presented OVA antigen on their MHC class I molecules (Fig. 2C), suggesting that the antigen introduced into the cytoplasm directly by JTT was processed into OVA peptides (OVA257-264 (SL8) SIINFEKL) (15). In this case, JTT had an $\mathrm{EC}_{50}$ of $133.7 \mu \mathrm{g} / \mathrm{ml}$ against antigen presentation to the MHC class I pathway in DC2. 4 cells. We next investigated whether JTT induced antigen presentation in primary dendritic cells as well as in dendritic cell lines. The antigen presentation of primary dendritic cells was also enhanced by JTT (Fig. 3).

On the other hand, JTT was composed of ten medical herbals. Of the 10 medical herbal ingredients, peony root and poria sclerotium have the strongest effects on increasing antigen presenting ability (Table I).

No cytotoxicity in DC2.4 cells was observed by treatment with JTT and the 10 medical herbals, except for cinnamon bark at $200 \mu \mathrm{g} / \mathrm{ml}$ (data not shown).

Tumor regression by the combination of OVA vaccination and $J T T$. To clarify the efficacy of JTT as an adjuvant for tumor vaccines, mice immunized with OVA were intradermally 
A

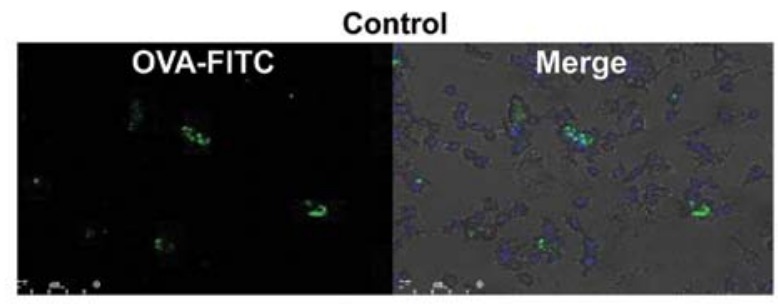

B

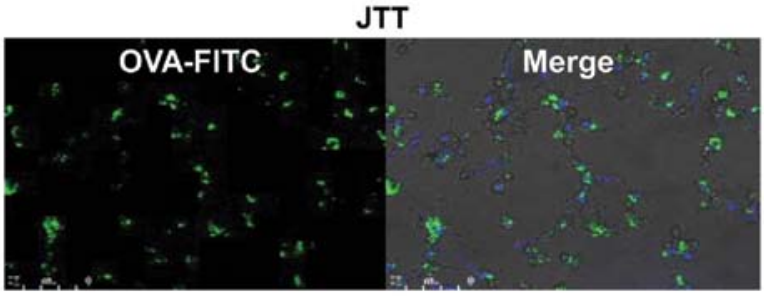

C

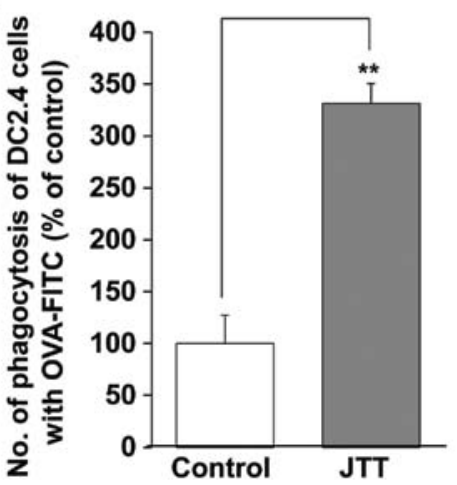

D

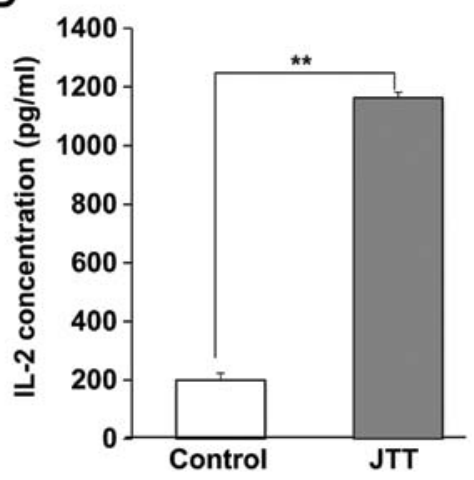

Figure 2. JTT increase antigen phagocytosis and antigen presentation to MHC class I pathway in DC2.4 cells in vitro. DC2.4 cells were exposed with OVA-FITC in the absence (A) and presence (B) of JTT for $20 \mathrm{~h}$. Phagocytic OVA antigens were detected with FITC (green) and nuclei were stained with DAPI (blue) by immunofluorescence microscopy. The numbers of DC2.4 cells taking OVA was calculated by NIH Image J. (C) Similar results were obtained in three independent experiments. DC2.4 cells preincubated with JTT cells were exposed to OVA for $2 \mathrm{~h}$. (D) IL-2 produced from CD8-OVA1.3 cells was stimulated with DC2.4 cells for the antigen presentation assay to the MHC class I pathway. Data are the mean $\pm \mathrm{SD}(\mathrm{n}=3)$; $\mathrm{P}<0.05,{ }^{* *} \mathrm{P}<0.01$, compared with the control.
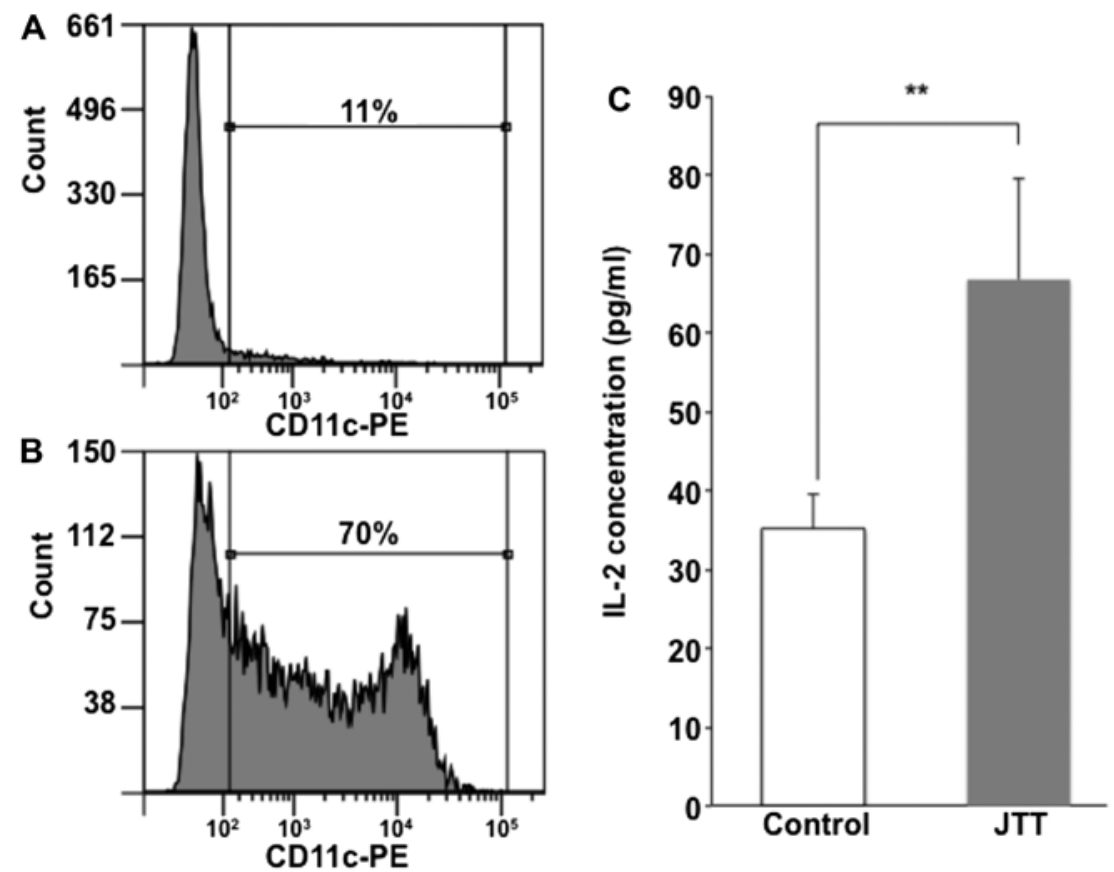

Figure 3. JTT increase antigen phagocytosis and antigen presentation to MHC class I pathway in primary DC2.4 cells in vitro. (A and B) Isolation of CD11c positive cells from mouse spleens. Mouse lymphocytes isolated from spleens and CD11c positive cells were selected using magnetic-activated cell sorting (MACS, Miltenyi Biotec, Gladbach, Germany). The cells before MACS selection (A) and after selection (B) were stained with CD11c antibodies conjugated to PE and analyzed by flow cytometry using a FACSCantoII system. (C) Antigen cross-presentation assays using CD11c positive cells isolated from mouse spleens. CD11c positive cells were incubated with OVA/LPF solution with $0-200 \mu \mathrm{g} / \mathrm{ml}$ of JTT and assayed for antigen cross-presentation as described in Materials and methods. The data are representative of two independent experiments with similar results. The data are expressed as mean $\pm \mathrm{SD}(\mathrm{n}=3)$. ${ }^{*} \mathrm{P}<0.05$. 
Table I. Effects of ten medical herbals on antigen presentation to MHC class I pathway in DC2.4 cells in vitro.

Herbal

Percentage of control $(\%)$

Astragalus root

98.0

Cinnamon bark

25.1

Rehmannia root

123.5

Peony root

282.3

Cnidium rhizome

175.5

Japanese angelica root

236.9

Ginseng

131.6

Poria sclerotium

313.8

Glycyrrhiza

183.4

Atractylodes lancea rhizome

176.6

DC2.4 cells were incubated with the ten herbal ingredients found in JTT $(100 \mu \mathrm{g} / \mathrm{ml})$ or water as a control at $37^{\circ} \mathrm{C}$ for $1 \mathrm{~h}$ and then incubated with OVA for $20 \mathrm{~h}$. The cells were cultured with CD8-OVA1.3 cells for $24 \mathrm{~h}$. The response of stimulated CD8-OVA1.3 cells was determined by their IL-2 levels. Data are presented as $\%$ of control.

challenged with E.G7-OVA tumor expressing the SL8 epitope as the model tumor antigen. Oral administration of JTT alone did not generate protective immunity against tumor growth, the same as the control, whereas tumor growth was inhibited by OVA vaccine (Fig. 4A and B). Together with JTT, OVA vaccine markedly inhibited the growth of E.G7-OVA tumor.
Consequently, tumor volume significantly decreased to $<40 \%$, comparing the combination of OVA vaccine and JTT to OVA vaccine alone. While no mouse immunized with OVA vaccine alone completely rejected E.G7-OVA tumor formation, the combination of OVA vaccine and JTT resulted in 2 of 10 mice exhibiting complete rejection, and prolonged survival was observed in mice treated with the combination compared to OVA vaccine alone (Fig. 4C).

Antitumor effect by immunological modulation by JTT. To verify the immune adjuvant effects of JTT, humoral and cellular immune responses to the OVA vaccine were measured. The humoral immune responses specific to the OVA vaccine were investigated by OVA antigen-specific IgG in mouse serum by ELISA. OVA antigen-specific IgG2b in the OVA and JTT mouse group was markedly increased $\sim 6$-fold compared to the OVA alone group (Fig. 5A), and compared to OVA antigenspecific IgG1 (data not shown). T-cell responses to the vaccine were measured by the IFN $-\gamma$ ELISPOT assay with splenocytes isolated from mice immunized with OVA with oral administration of JTT. OVA antigen-specific IFN- $\gamma$-secreting CD8 $\mathrm{T}$ cells significantly increased $\sim 3$-fold compared to the OVA vaccine alone mouse group with OVA and with oral administration of JTT (Fig. 5B).

\section{Discussion}

There are a number of reports that some Kampo medicine activates innate immunity. JTT is often used clinically in tumor patients who have received chemotherapy or radiation therapy to improve anorexia or remission of leucopenia (Fig. 1).

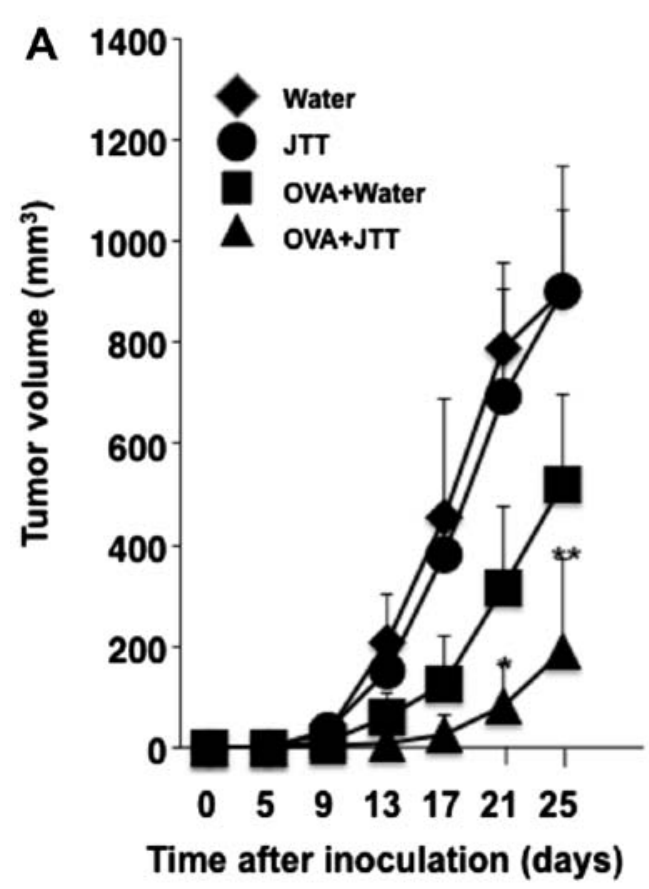

B
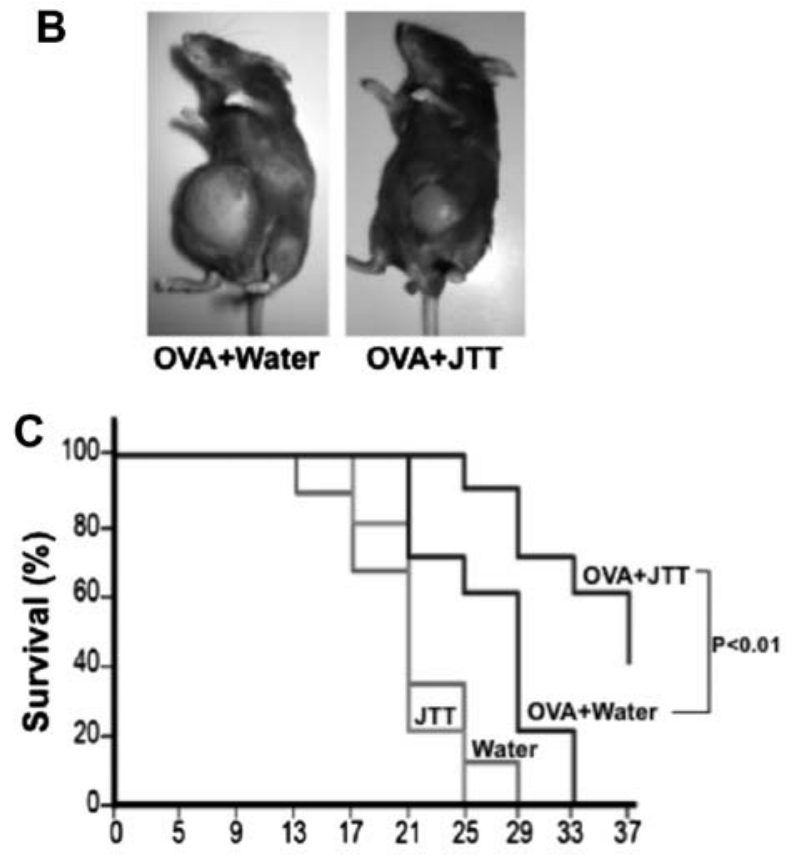

Time after inoculation (days)

Figure 4. Adjuvant effect against tumor vaccine of JTT in tumor-bearing mice. JTT or water was orally administered to 10 mice from day 7 to day 37 consecutively. Seven days after immunization (day 0), mice were inoculated intradermally with OVA-expressing EG7 cells $\left(5 \times 10^{5}\right)$. (A) Tumor volume was measured every 4 days and tumor volume was calculated. Data are the mean $\pm \mathrm{SD}(\mathrm{n}=10)$; ${ }^{*} \mathrm{P}<0.05,{ }^{* * *} \mathrm{P}<0.01$, compared with the control. (B) Tumor-bearing mice 25 days after tumor inoculation. (C) Overall survival was estimated by Kaplan-Meier analysis and compared using log-rank tests ( $\mathrm{P}=0.0032$, OVA vs OVA + JTT). 

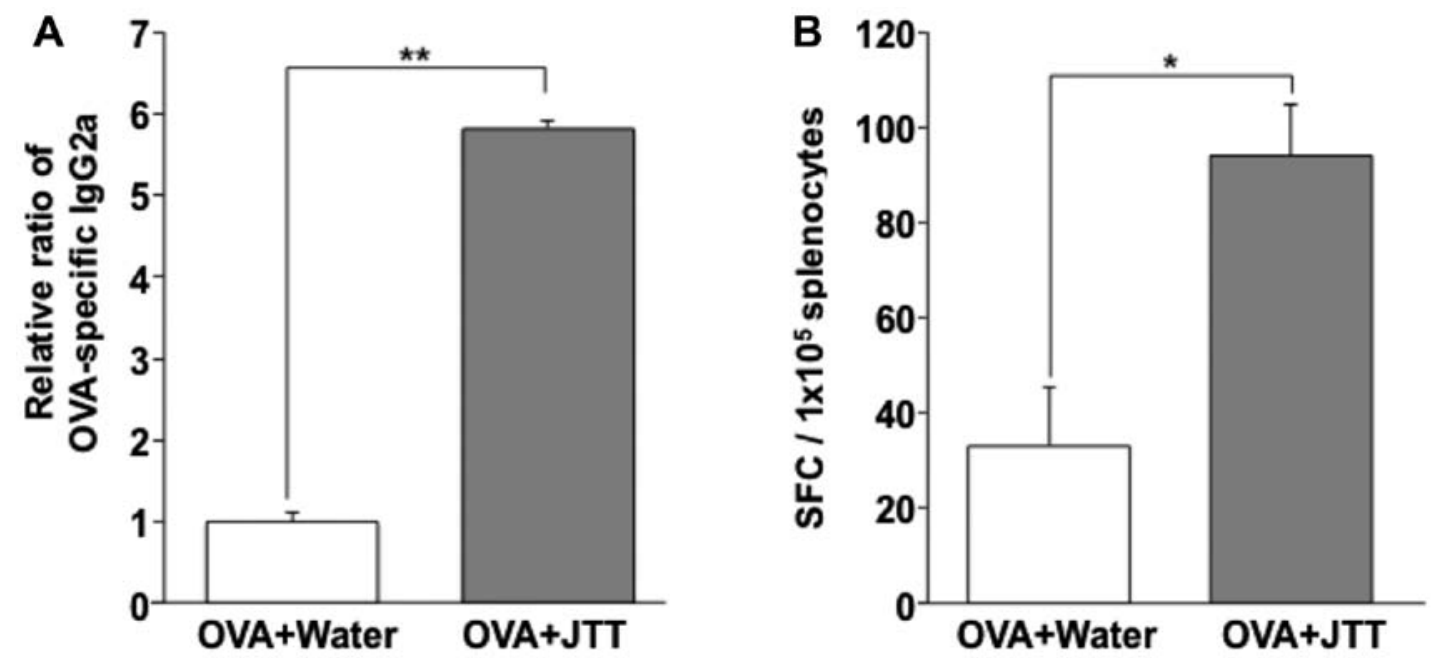

Figure 5. JTT enhances cellular and humoral immune responses to the OVA vaccine in vivo. One group was administered JTT ( $2 \mathrm{~g} / \mathrm{kg} / \mathrm{day})$ and the other was administered water once a day. OVA + Water group: water was orally administered to 3 mice from day 7 to day 14 consecutively. In addition, these mice were immunized with OVA on day 7 and day 0 twice. OVA + JTT group: JTT was orally administered to 3 mice from day 7 to day 14 consecutively. In addition, these mice were immunized with OVA on day 7 and day 0 twice. On day 14, mice of this group were sacrificed, and the splenocytes and serum were collected. Splenocytes were re-stimulated with OVA $(10 \mu \mathrm{g} / \mathrm{ml})$. (A) OVA-specific IgG2a in mouse serum immunized with OVA with or without the administration of JTT or water was determined by ELISA. The relative ratio of OVA-specific IgG2a is shown comparing the OVA + JTT group with the OVA + Water group. Each group contained 3 mice and all experiments were repeated 3 times. Data are the mean $\pm \mathrm{SD} ;{ }^{*} \mathrm{P}<0.05$, compared with the control (OVA + Water) group. (B) Data are expressed as spot-forming IFN- $\gamma$-secreting T cells (SFC) responding to OVA by ELISPOT assay (SFC/1x10 ${ }^{5}$ splenocytes). Each group contained 3 mice and all experiments were repeated 3 times. Data are the mean $\pm \mathrm{SD} ;{ }^{*} \mathrm{P}<0.05,{ }^{* *} \mathrm{P}<0.01$, compared with the control (OVA + Water) group.

It has been reported that JTT can enhance natural killer (NKT) cell-inducing activity and the ability to produce antibodies in the liver (1). Ohnishi et al reported that liver metastasis was suppressed by oral administration of JTT to mice and its efficacy was induced by macrophages and $\mathrm{T}$ cells $(5,6)$. In addition, as reported by Chino et al, JTT enhanced LPS-induced IL-12 and IFN- $\gamma$ production via toll-like receptor (TLR) in macrophages (7). Thus, JTT is considered to be a drug with potential as a biological response modifier (BRM), boosting its efficacy and activating innate immunity.

However, while its role as a BRM is expected, the effect of Kampo on the acquired immune system, such as antigenspecific immune induction, i.e., vaccines, remains unclear. In order to induce tumor antigen-specific immune responses using tumor-associated antigens inoculated as vaccines, first of all, the antigen needs to be taken up by an antigen-presenting cells (APC), such as dendritic cells. Then, in the cytoplasm of APC, an epitope peptide fragmented by vaccine antigens needs to be presented to MHC class I, which are cell membrane surface molecules in APC (16).

In this study, we examined whether JTT can induce a vaccine antigen-specific immune response, and whether it can be applied as a tumor vaccine adjuvant.

Firstly, the effect of JTT on the phagocytic ability of a vaccine model antigen (OVA) in dendritic cells was investigated (Fig. 2). We also examined the impact of JTT on the antigen-presenting ability of dendritic cell lines (DC2.4 cells) and primary dendritic cells to $\mathrm{T}$ cells using IL-2 production from MHC class I and OVA complex-restricted T cell clones as an indicator. As a result, JTT strongly enhanced the antigen-presenting ability of both DC2.4 (Fig. 2C) and primary dendritic cells (Fig. 3C). From these findings, the possibility that JTT has an adjuvant effect on vaccines was suggested. Therefore, we next examined the efficacy of JTT as an adjuvant for tumor vaccines using inoculated EG7 mouse tumors presented with tumor model antigen (OVA) $(17,18)$.

As a result, despite the failure to observe antitumor effects from the administration of JTT alone, we were able to observe that inoculation of OVA vaccine allowed the induction of antitumor specific immunity against EG7. We also observed that the combined use of JTT inhibited tumor growth significantly and prolonged the tumor engraftment period as well as the survival period (Fig. 4). These findings confirmed that JTT has adjuvant efficacy for tumor vaccines. In order to demonstrate the efficacy of JTT as an adjuvant, we analyzed the vaccine antigen-specific immune induction mechanism (vaccine antigen-specific IgG, IFN- $\gamma$-secreting CD8 T cells, and CTL).

It is known that IL-4 of Th2-type cytokines is involved in class switching to IgG1 from IgM, while cytokine IFN- $\gamma$ of Th1 type is involved in class switching to $\operatorname{IgG} 2 \mathrm{a}(19,20)$. IFN- $\gamma$ is a cytokine involved in the induction of tumor-specific CTL, and IFN- $\gamma$ produced from Th1 cells tilts the Th1/Th2 balance to Th1 dominantly (21).

As a result, we observed the significant production of OVA-antigen specific IgG2a and IFN- $\gamma$-secreting CD8 T cells by the combination of OVA vaccine and oral administration of JTT (Fig. 5). In addition, the tendency of increasing of OVA antigen-specific CTL (cytotoxic T lymphocytes) by JTT could be observed using MHC tetramer by FACS (data not shown). Further study will focus on the CTL for clearing of the antitumor mechanism of JTT.

For the future development of novel adjuvants from Kampo formulas, it is important to identify the active compounds derived from medical herbal components, which have remarkable vaccine antigen-specific immune responses. As shown in Fig. 1, JTT consists of ten medical herbal ingredients (astragalus root, cinnamon bark, rehmannia root, peony root, cnidium rhizome, Japanese angelica root, ginseng, poria sclerotium, 
glycyrrhiza and Atractylodes lancea rhizome). In DC.2.4 cells, peony root and poria sclerotium were found to have the strongest effect on increasing antigen presenting ability of the ten medical herbs (Table I). We will thus try to identify an active compound with this ability in the two medical herbs.

In wake of the discovery of the tumor antigen (22), tumor vaccine therapy has drawn attention, with attempts to obtain an antitumor effect by inducing tumor antigen-specific immune responses in the host.

However, despite the current progress, in which useful tumor antigens have been identified and the whole aspect of the immune system surrounding tumors is being elucidated, tumor vaccine therapy is not satisfactory compared with other treatments such as chemotherapy. To improve the therapeutic effect, various approaches have been attempted, including the development of vaccine materials $(8,10)$.

Here we focused on Japanese traditional herbal medicine (Kampo) as an immune adjuvant for tumor vaccine. Currently, Kampo is used in a number of clinical situations such as cancer therapy, and contributes to health care (23-28).

Therefore, it is extremely important to confirm the enhancement of the antitumor effect by combining Kampo with chemotherapy using existing antitumor agents or tumor vaccine treatments which have been carried out in clinical practice.

On the other hand, OVA was used as a tumor model antigen in this study. For this reason, in order to introduce the combined use of Kampo for tumor vaccine treatment into clinical practice, in our future key studies it will be necessary to examine the potential efficacy of JTT as an adjuvant by using an actual tumor-related antigen, and to explore better Kampo drugs than JTT to serve as more potent adjuvants.

\section{Acknowledgements}

This study was supported by a Grant-in-Aid for Health Labour Sciences Research Grant and a Grant-in-Aid for the Cooperative Research Project from Institute of Natural Medicine, University of Toyama. We thank Dr Tsuyoshi Nakanishi (Laboratory of Hygienic Chemistry and Molecular Toxicology, Department of Biopharmaceutical Sciences, Gifu Pharmaceutical University, Japan), Dr Takeshi Yamamoto, Dr Makoto Kadowaki (Division of Gastrointestinal Pathophysiology, Institute of Natural Medicine of Toyama, Japan) and Dr. Kazuo Ogawa (Tsumura Research Laboratories) for helpful advice and technical support.

\section{References}

1. Matsumoto T, Sakurai MH, Kiyohara H and Yamada H: Orally administered decoction of Kampo (Japanese herbal) medicine, 'Juzen-Taiho-To' modulates cytokine secretion and induces NKT cells in mouse liver. Immunopharmacology 46: 149-161, 2000.

2. Kiyohara H, Matsumoto T and Yamada H: Combination effects of herbs in a multi-herbal formula: Expression of Juzen-taiho-to's immuno-modulatory activity on the intestinal immune system. Evid Based Complement Alternat Med 1: 83-91, 2004.

3. Kiyohara H, Matsumoto T, Takemoto N, Kawamura H, Komatsu Y and Yamada H: Effect of oral administration of a pectic polysaccharide fraction from a Kampo (Japanese herbal) medicine 'juzen-taiho-to' on antibody response of mice. Planta Med 61: 429-434, 1995.

4. Iijima K, Sun S, Cyong JC and Jyonouchi H: Juzen-taiho-to, a Japanese herbal medicine, modulates type 1 and type $2 \mathrm{~T}$ cell responses in old BALB/c mice. Am J Chin Med 27: 191-203, 1999.
5. Ohnishi Y, Fujii H, Hayakawa Y, Sakukawa R, Yamaura T, Sakamoto T, Tsukada K, Fujimaki M, Nunome S, Komatsu Y, et al: Oral administration of a Kampo (Japanese herbal) medicine Juzen-taiho-to inhibits liver metastasis of colon 26-L5 carcinoma cells. Jpn J Cancer Res 89: 206-213, 1998.

6. Onishi Y, Yamaura T, Tauchi K, Sakamoto T, Tsukada K, Nunome S, Komatsu Y and Saiki I: Expression of the antimetastatic effect induced by Juzen-taiho-to is based on the content of Shimotsu-to constituents. Biol Pharm Bull 21: 761-765, 1998

7. Chino A, Sakurai H, Choo MK, Koizumi K, Shimada Y, Terasawa K and Saiki I: Juzentaihoto, a Kampo medicine, enhances IL-12 production by modulating Toll-like receptor 4 signaling pathways in murine peritoneal exudate macrophages. Int Immunopharmacol 5: 871-882, 2005.

8. Munekage M, Ichikawa K, Kitagawa H, Ishihara K, Uehara H, Watanabe J, Kono T and Hanazaki K: Population pharmacokinetic analysis of daikenchuto, a traditional Japanese medicine (Kampo) in Japanese and US health volunteers. Drug Metab Dispos 41: 1256-1263, 2013.

9. Xie Y, Jiang ZH, Zhou H, Ma WZ, Wong YF, Liu ZQ and Liu L: The pharmacokinetic study of sinomenine, paeoniflorin and paeonol in rats after oral administration of a herbal product Qingfu Guanjiesu capsule by HPLC. Biomed Chromatogr 28: 1294-1302, 2014.

10. Chen Y, Ma Y and Ma W: Pharmacokinetics and bioavailability of cinnamic acid after oral administration of Ramulus Cinnamomi in rats. Eur J Drug Metab Pharmacokinet 34: 51-56, 2009.

11. Zhao W-J, Wang BJ, Wei CM, Yuan GY, Bu FL and Guo RC: Determination of glycyrrhetic acid in human plasma by HPLC-MS method and investigation of its pharmacokinetics. $\mathbf{J}$ Clin Pharm Ther 33: 289-294, 2008.

12. Aranda F, Vacchelli E, Eggermont A, Galon J, Sautès-Fridman C, Tartour E, Zitvogel L, Kroemer G and Galluzzi L: Trial Watch: Peptide vaccines in cancer therapy. OncoImmunology 2: e26621, 2013.

13. Saiki I, Koizumi K, Goto H, Inujima A, Namiki T, Raimura M, Kogure T, Tatsumi T, Inoue H, Sakai S, et al: The long-term effects of a Kampo medicine, juzentaihoto, on maintenance of antibody titer in elderly people after influenza vaccination. Evid Based Complement Alternat Med 2013: 568074, 2013.

14. Reed SG, Orr MT and Fox CB: Key roles of adjuvants in modern vaccines. Nat Med 19: 1597-1608, 2013.

15. Bacik I, Cox JH, Anderson R, Yewdell JW and Bennink JR: TAP (transporter associated with antigen processing)-independent presentation of endogenously synthesized peptides is enhanced by endoplasmic reticulum insertion sequences located at the amino- but not carboxyl-terminus of the peptide. J Immunol 152: 381-387, 1994.

16. Harding CV: Class I MHC presentation of exogenous antigens. J Clin Immunol 16: 90-96, 1996.

17. Babu JS, Nair S, Kanda P and Rouse BT: Priming for virusspecific $\mathrm{CD}^{+}$but not $\mathrm{CD}^{+}$cytotoxic $\mathrm{T}$ lymphocytes with synthetic lipopeptide is influenced by acylation units and liposome encapsulation. Vaccine 13: 1669-1676, 1995.

18. Takahashi H, Takeshita T, Morein B, Putney S, Germain RN and Berzofsky JA: Induction of $\mathrm{CD}^{+}$cytotoxic $\mathrm{T}$ cells by immunization with purified HIV-1 envelope protein in ISCOMs. Nature 344: 873-875, 1990.

19. Snapper CM and Paul WE: Interferon-gamma and B cell stimulatory factor- 1 reciprocally regulate Ig isotype production. Science 236: 944-947, 1987.

20. Xu Z, Zan H, Pone EJ, Mai T and Casali P: Immunoglobulin class-switch DNA recombination: Induction, targeting and beyond. Nat Rev Immunol 12: 517-531, 2012.

21. Gajewski TF and Fitch FW: Anti-proliferative effect of IFN-gamma in immune regulation. I. IFN-gamma inhibits the proliferation of Th2 but not Th1 murine helper T lymphocyte clones. J Immunol 140: 4245-4252, 1988.

22. van der Bruggen P, Traversari C, Chomez P, Lurquin C, De Plaen E, Van den Eynde BJ, Knuth A and Boon T: A gene encoding an antigen recognized by cytolytic $\mathrm{T}$ lymphocytes on a human melanoma. J Immunol 178: 2617-2621, 2007.

23. Uezono Y, Miyano K, Sudo Y, Suzuki M, Shiraishi S and Terawaki K: A review of traditional Japanese medicines and their potential mechanism of action. Curr Pharm Des 18: 4839-4853, 2012. 
24. Yamakawa J, Motoo Y, Moriya J, Ogawa M, Uenishi H, Akazawa S, Sasagawa T, Nishio M and Kobayashi J: Significance of Kampo, traditional Japanese medicine, in supportive care of cancer patients. Evid Based Complement Alternat Med 2013: 746486, 2013.

25. Okumi $\mathrm{H}$ and Koyama A: Kampo medicine for palliative care in Japan. Biopsychosoc Med 8: 6, 2014.

26. Suzuki H, Asakawa A, Amitani H, Fujitsuka N, Nakamura N and Inui A: Cancer cachexia pathophysiology and translational aspect of herbal medicine. Jpn J Clin Oncol 43: 695-705, 2013.

27. Iwase S, Yamaguchi T, Miyaji T, Terawaki K, Inui A and Uezono Y: The clinical use of Kampo medicines (traditional Japanese herbal treatments) for controlling cancer patients symptoms in Japan: A national cross-sectional survey. BMC Complement Altern Med 12: 222, 2012.

28. Mantani N, Kasahara Y, Kamata T, Sekiya N, Shimada Y, Usuda K, Sakakibara I, Hattori N and Terasawa K: Effect of Seihai-to, a Kampo medicine, in relapsing aspiration pneumonia - an open-label pilot study. Phytomedicine 9: 195-201, 2002.
29. Hayashi A, Wakita H, Yoshikawa T, Nakanishi T, Tsutsumi Y, Mayumi T, Mukai Y, Yoshioka Y, Okada N and Nakagawa S: A strategy for efficient cross-presentation of CTL-epitope peptides leading to enhanced induction of in vivo tumor immunity. J Control Release 117: 11-19, 2007.

30. Kato S, Koizumi K, Yamada M, Inujima A, Takeno N, Nakanishi T, Sakurai H, Nakagawa S and Saiki I: A phagocytotic inducer from herbal constituent, pentagalloylglucose enhances lipoplex-mediated gene transfection in dendritic cells. Bio Pharm Bull 33: 1878-1885, 2010.

31. Nakanishi T, Hayashi A, Kunisawa J, Tsutsumi Y, Tanaka K, Yashiro-Ohtani Y, Nakanishi M, Fujiwara H, Hamaoka T and Mayumi T: Fusogenic liposomes efficiently deliver exogenous antigen through the cytoplasm into the MHC class I processing pathway. Eur J Immunol 30: 1740-1747, 2000. 